light, make the stone a remàrkable freak of nature; and not a little interest is added to it by the fact that its strange features were so well developed by mere accident.

Among curiously marked diamonds resembling the above, may also be mentioned the two presented to the Jardin des plantes by Malphen, and described by Descloizeaux (see Poggendorff's annalen, 1849, ix. p. 447).

These stones are colorless and round, and a distinct three-leaved clover in black occupies the entire dimension in each stone. Another in the Duke of Leuchtenberg's cabinet, now in the Bavarian state cabinet at Munich, has three leaves united by a circle. All these three are of Indian origin.

A curious diamond (fig. 6), also in the possession of Messrs. Tiffany and Co., is a red brilliant, that at first glance appears brown, while through it a beautiful dark rose-red light breaks in every direction : really, therefore, it is a red brilliant, or combination of red and brown; or, more correctly, a red diamond with a brown cloud, the red predominating as the stone is turned, or the light strikes it in different directions. This change of color gives the stone its dichroitic effect, although no effect is produced in viewing it by the dichroscope.

By artificial light it appears brown; but the entire stone throws ont bright red reflections, such as are produced by the diamond only.

One-half of the stone is filled with hundreds of irregular-shaped cavities, either empty, or filled with a transparent fluid, or, as in nearly all cases, with carbon, which in some instances is in pieces, or so fractured as to admit the light through it. 'These inclusions appear to affect the color sufficiently to produce the brown appearance.

Light seems to be very faintly restored under the crossed Nichols prisms, in addition to that produced by the cutting of the gem. The specific gravity is 3.5696. George F. Kunz.

\section{THE DISTRIBUTION OF COMETS WITH REFERENCE TO SOLAR MOTION.}

THE regions of space outside the solar system furnish a supply of comets which seems to be inexhaustible. Their origin is simply a matter for speculation; and it is not reasonably to be expected, that, even with the large amount of attention being given to cometary investigation, any definite information upon this subject can be attained for centuries to come. There is no probability that any considerable number of them have had their origin in the mass which has gone to form the solar system.
Between six and seven hundred comets have been recorded; and if we take the large proportion of telescopic comets which have been discovered when searching has been vigorously prosecuted, to the number visible without the aid of a telescope, it would not be an exaggerated estimate to place the number of comets which have come into our system since the beginning of the Christian era, under such conditions as to have been visible by the aid of our modern appliances, at four thousand.

Undoubtedly there are many, which, either from their small size and faintness, or from the unfavorable positions of their orbits, never could be seen. Were the orbits of all these known, the discussion would furnish some reasonable basis for an hypothesis in regard to the origin of the comets themselves ; and light would be thrown upon other problems concerning the system of the universe.

While the sun, drawing all bodies within its influence towards itself, swings the comets in to orbits which may bring them, at some point, almost into contact with the sun's surface, or, on the other hand, never within the confines of our planetary system at all, the motion of the sun itself must have an influence upon the position of these orbits. The limited amount of material at hand furnishes some features which are at least suggestive.

There are two hundred and eighty-five comets, which, up to the present time, have had their orbits determined with greater or less exactness. The axes of the orbits lie in the directions along which the comets come to the sun.

The table exhibits the grouping of the points which represent the directions of the origins of these comets about that point which investigation has indicated as the direction to which the solar system is moving.

The method of making the reductions will be apparent. The elements were reduced to 1850 , and the longitude and latitude of the perihelion point computed for each comet. The point directly opposite this was then reduced to a new system of co-ordinates, having the direction of solar motion, as the pole.

The point toward which the sun is moving is taken at longitude $256^{\circ}$, latitude $+57^{\circ}$.

For convenience this may be referred to as the north pole of the new system; and the hemisphere surrounding it, as the northern. This hemisphere is then divided into ten zones of equal area, the most northern surrounding the pole, while that at the base may be called the equatorial.

The southern hemisphere is similarly divid- 
ed, and in the table the figures in the top line are for the north and south polar zones.

Those adjoining each are given next, so that the lower line contains the zones immediately north and south of the equator. Zones on the same line are at the same distance from the north and south poles respectively.

The columns give the number of comets whose points of apparent origin fall in each zone, and the mean of the peribelion distances of the group.

\begin{tabular}{r|c||c|c}
\hline \multicolumn{2}{c|}{ NorTH. } & \multicolumn{2}{|c}{ Soutu. } \\
\hline No. & $\begin{array}{c}\text { Mean peribelion } \\
\text { distance. }\end{array}$ & No. & $\begin{array}{c}\text { Mean perihelion } \\
\text { distance. }\end{array}$ \\
\cline { 2 - 3 } 9 & 0.730 & 16 & 0.599 \\
8 & 1.022 & 13 & 0.713 \\
13 & 0.745 & 14 & 0.715 \\
12 & 0.700 & 13 & 0.526 \\
15 & 0.838 & 28 & 0.795 \\
14 & 1.010 & 11 & 1.008 \\
20 & 0.821 & 17 & 0.813 \\
11 & 0.903 & 10 & 1.091 \\
19 & 0.767 & 15 & 0.789 \\
15 & 0.762 & & 0.912 \\
\hline
\end{tabular}

So many conditions that will readily occur to any one come in to affect the number of comets discovered, and, to a certain extent, the discovery of those coming from any particular quarter, that the collection of so small a proportion cannot give much satisfaction. It will be noticed that the zones farthest south have a larger number than the corresponding zones north, while near the equator this difference is reversed.

The mean perihelion distances, taking the columns separately, vary in such a way that there is little encouragement for discussion as a whole.

But taking the corresponding zones in the two hemispheres, the comparison is interesting, if not instructive. The first seven from the north pole, or direction of solar motion, downward, have greater mean perihelion distances than those in the southern hemisphere similarly situated. The equatorial zones, where the distinction would not be so great, have an opposite difference.

With the same data combined in other proportions, the differences will be found to confirm the tendency shown by this division. 'Thus doubling the area of the zones, making five northern and five southern, the excess of mean perihelion distances of the north over the south exists in the three polar sets. Comparisons can also be made by fours and fives, and also by combining the two adjacent to the polar zones, the three next following these, and finally the four next north and south of the equator. Any one can make these comparisons with small uncertainty, from the table.

The most satisfactory confirmation of the tendencies here indicated is found in the discussion of the comets of the last hundred years only. These have been well observed in general, and the number does not contain so large a proportion of anomalous orbits. The table above includes all, probably, that have been computed; but, in summing up, notice was taken of the effect of the unusual cases, like the large perihelion distance of the 1729 comet, and the combination of several very small distances in one zone; and in no case would the sign of the compared difference have been changed by the omission of any extraordinary comets.

For the one hundred years the numbers in the zones are more uniform, with a similar tendency to that above.

In the comparison of perihelion distances by zones, seven of the northern exceed the corresponding southern.

The general results may be summed up in a few lines. There is an indication that more comets come in from the hemisphere from which the sun is moving.

The zones in the hemisphere towards which the sun is moving, and which has for its pole the direction of solar motion, have in general greater perihelion distances than the corresponding zones in the other hemisphere; the tendency being best exhibited as we go from the equator of the system.

As the sun moves on, the comets at great distances would come into the system, eventually, behind the quarter in which they first yielded to the attraction. Under the same general conditions, those which have come from behind the sun, and have been, as it were, dragged in its train, would pass nearest to the point of attraction when overtaking it.

These are the suggestions which most naturally occur. A complete discussion of the effect of the solar motion upon the distribution of comet-origins can hardly receive any decided confirmation with the amount of material that is likely to be available for generations to come.

R. H. 'TUCKer, Jun.

Lehigh university.

VOLCANIC SAND WIICH FELL AT UNALASHKA, ALASKA, OCT. 20, 1883, AND SOME CONSIDERATIONS CONCERNING ITS COMPOSITION.

Mr. Applegate, the signal-service observer at Unalashka, reports that on the 20th of 